\title{
Amélioration de la tenue à la corrosion atmosphérique des matériaux utilisés en connectique. Traitements de surface par laser
}

\author{
C. Georges, C. Perrin, D. Simon, F. Fariaut ${ }^{1}$ et C. Boulmer-Leborgne ${ }^{1}$
}

CERI, CNRS, $3 A$ rue de la Férollerie, 45071 Orléans cedex 2, France

1 GREMI, UMR CNRS, Université d'Orléans, BP. 6744, 45067. Orléans cedex 2, France

\begin{abstract}
Résumé : L'industrie des composants passifs occupe une place importante dans la filière électronique (télécommunications, automobile, informatique...). Dans ce secteur d'activités, la connectique constitue la part la plus importante. Les contacts électriques sont constitués d'un alliage de cuivre (laiton ou bronze) recouvert de nickel (barrière de diffusion) et d'or. Il existe des défauts d'étanchéité dans les couches d'or et de nickel qui induisent la corrosion des couches sous-jacentes. Afin de modifier la structure du dépôt d'or, des traitements de surface par laser UV ont été entrepris car l'absorption de l'or est meilleure dans l'UV. Ils ont pour objectif de supprimer les porosités de la couche d'or à l'origine des points de corrosion et d'effectuer un lissage de la surface car la rugosité est nuisible à un bon contact électrique.
\end{abstract}

\section{INTRODUCTION}

De nombreuses études ont déjà été réalisées dans le domaine de la corrosion atmosphérique des contacts électriques à bas niveau de courant. Les matériaux se présentent sous forme de plaquettes que nous traitons dans des atmosphères corrosives constituées d'air synthétique humide contenant différents polluants à faibles concentrations (dioxyde d'azote, dioxyde de soufre, hydrogène sulfuré et dichlore). Pour ces substrats dorés, les tests de corrosion déjà effectués ont montré que la cause principale de la corrosion est la présence de défauts d'étanchéité dans les couches d'or et de nickel.

Le but des traitements laser envisagés pour remédier à ce problème est double : effectuer un lissage de la surface et supprimer les porosités de la couche d'or. En effet, la rugosité et les porosités sont liées à la croissance colonnaire des dépôts. La rugosité est nuisible à un bon contact électrique. Les porosités sont à l'origine des points de corrosion.

\section{RESULTATS}

Les premiers essais, ont été effectués avec un laser à excimère $\mathrm{XeCl}(\lambda=308 \mathrm{~nm})$ [1]. Ce traitement avait pour but de fondre le dépôt d'or (d'épaisseur $1 \mu \mathrm{m}$ ) en surface. Ce traitement de lissage de la couche d'or a été suivi d'un test de corrosion. Les résultats obtenus sont assez concluants. Le lissage de la couche est très net (figure 1) et le test de corrosion effectué à un taux d'humidité de $90 \%$ montre une amélioration de la résistance à la corrosion dans les zones traitées laser (figure 2).

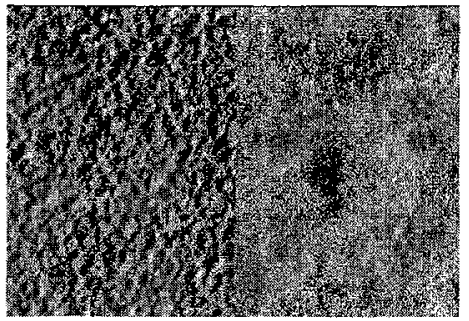

figure 1

Comparaison de l'état de surface d'un dépôt d'or de 1 $\mu \mathrm{m}$ -non traitée à gauche- après traitement laser à droite (Photo en mode rétrodiffusé $\mathbf{G}:$ x 10 000)

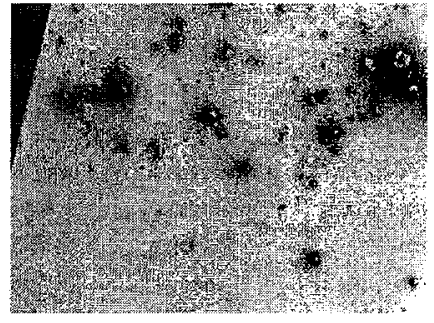

figure 2

Zones traitées laser et non traitées, après corrosion dans de ''air synthétique humide contenant $\mathrm{NO}_{2}(0,2 \mathrm{pm}), \mathrm{SO}_{2}$ $(0,2 \mathrm{vpm}), \mathrm{Cl}_{2}(0,01 \mathrm{vpm}) ; \mathrm{HR}=90 \% ; \mathrm{t}=186 \mathrm{~h}$ 
Actuellement nous utilisons un laser à excimère Lambda physik Compex $205 \mathrm{KrF}(\lambda=0,248 \mu \mathrm{m})$ sur lequel est installé un homogénéiseur de faisceau. Les tests de corrosion effectués sur les échantillons traités montrent que dans les impacts comportant 5 ou 10 tirs laser, il y a nettement moins de germes que dans la zone non traitée (figures 3 ) mais les observations microscopiques montrent que l'état de surface a été modifié.

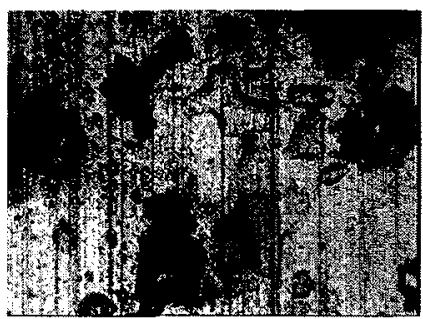

a) zone non traitée

figures 3

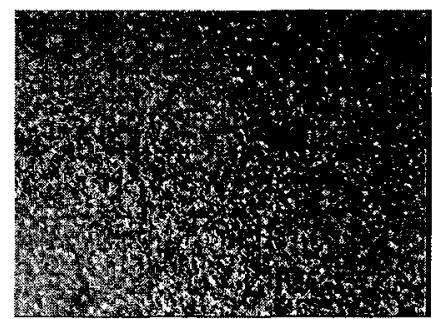

b) Impact laser ( 5 tirs)

Zones traitées laser et non traitées après corrosion dans de l'air synthétique humide contenant $\mathrm{NO}_{2}(0,2 \mathrm{pm}), \mathrm{SO}_{2}(0,2$ vpm), $\mathrm{Cl}_{2}(0,01$ vpm) $; \mathrm{HR}=85 \% ; \mathrm{t}=155 \mathrm{~h}$

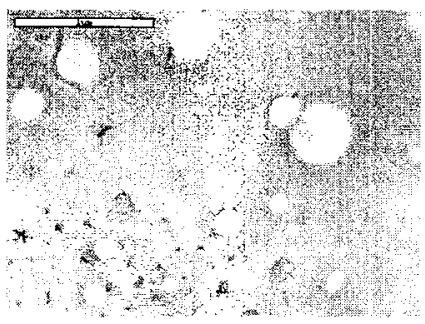

a) Impact laser (1 tix)

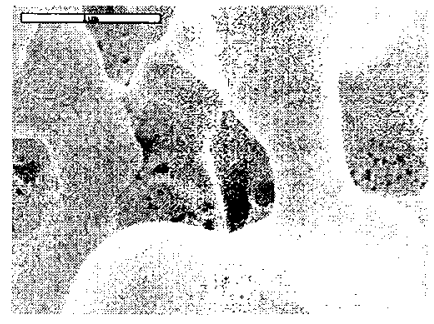

b) Impact laser (10 tirs)

figures 4

Etat de surface d'un dépôt d'or de $0,75 \mu \mathrm{m}$ après traitement laser (impacts avec 1 et 10 tirs) non corrodé (G: x 40 000)

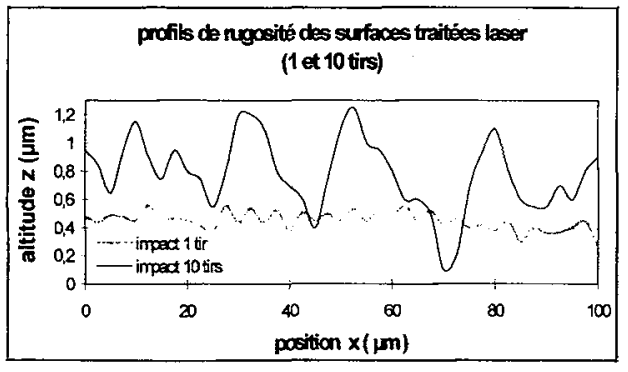

Les observations faites au MEB (Microscope Electronique à Balayage) à des grossissements de 40000 (figures 4) montrent que la surface est de plus en plus irrégulière au fur et à mesure que l'on augmente le nombre de tirs. L'aspect de la surface est différent entre la zone de l'impact 1 tir et celle de l'impact 10 tirs. Les profils de rugosité effectués dans ces impacts montrent que la rugosité augmente avec le nombre de tirs. Elle est caractéristique d'un chauffage de la surface trop important.

\section{CONCLUSION}

Les premiers essais réalisés ont montré que pour améliorer la résistance à la corrosion des contacts électriques, plusieurs tirs laser sont nécessaires. Néanmoins la rugosité dans ces impacts est plus forte que dans la zone non traitée. La fusion de la couche d'or est inhomogène.

Nous envisageons de faire des essais avec plusieurs tirs laser $(10,20,50,100$ tirs...) à des fluences beaucoup plus faibles pour espérer lisser la surface en réduisant la rugosité de la couche d'or déposée. En outre, nous allons effectuer ces essais en utilisant différentes longueurs d'ondes.

Ce travail est soutenu par la région Centre et la Société FCI (Framatome Connectors Internationals).

\section{Référence}

1. C. Perrin, D. Simon, J. Chim. Phys. 96, 1226-1244 (1999) et les références de cet article. 